\title{
Estudio Morfológico de Canales Nasopalatinos en Población Mexicana Mediante Tomografía Computarizada de Haz Cónico
}

\author{
Morphological Study of Nasopalatine Canal in Mexican \\ Population Using Cone Beam Computed Tomography
}

\begin{abstract}
Alejandro Rocha Castillo; José Obed García Cortes; Francisco Javier Gutiérrez Cantú; Alejandra Ruiz Flores; Abraham Israel Muñoz-Ruíz; José Carlos López Ramírez \& Jairo Mariel Cárdenas
\end{abstract}

ROCHA CASTILLO, A.; GARCÍA CORTES, J. O.; GUTIÉRREZ CANTÚ, F. J.; RUIZ FLORES, A.; MUÑOZ-RUÍZ, A. I.; LÓPEZ RAMÍREZ, J. C. \& MARIEL CÁRDENAS, J. Estudio morfológico de canales nasopalatinos en población mexicana mediante tomografía computarizada de haz cónico. Int. J. Morphol., 37(4):1272-1279, 2019.

RESUMEN: La morfología y dimensiones de ciertas estructuras anatómicas varían de población a población, así como de individuo a individuo; el canal nasopalatino (CNP) es una de estas estructuras, este se encuentra ubicado en la línea media del paladar y aloja el nervio nasopalatino y la rama terminal de la arteria nasopalatina. El propósito de este estudio es determinar la morfología y dimensiones promedio del CNP en la población mexicana mediante tomografía computarizada de haz cónico (CBCT). Se analizaron 120 CBCT de manera coronal, transversal y sagital; y se clasificaron siguiendo los parámetros de Bornstein. Para el análisis estadístico se determinó la normalidad de las variables empleando la prueba de Shapiro Wilk y la significancia estadística mediante la prueba de UMann Whitney. Los resultados mostraron diferencias estadísticas significativas en las variables analizadas del canal nasopalatino entre hombres y mujeres. De acuerdo con los datos obtenidos se puede establecer que la morfología del CNP es muy variable y se recomienda realizar un estudio morfológico y dimensional antes de cualquier intervención quirúrgica relacionada con esta zona.

PALABRAS CLAVE: Canal nasopalatino; Parámetros de Bornstein; Tomografía Computarizada de Haz Cónico.

\section{INTRODUCCIÓN}

El canal nasopalatino (CNP) contiene el nervio nasopalatino y la rama terminal de la arteria nasopalatina descendente, de igual manera presenta tejido conjuntivo fibroso, graso y glándulas salivales pequeñas, por lo que desde el punto de vista anatómico y quirúrgico es una zona muy relevante (Liang et al., 2009). El CNP se encuentra frecuentemente ubicado en la línea media del paladar y posterior a los incisivos centrales del maxilar (Jacobs et al., 2007); presenta una abertura oral en forma de embudo sobre la línea media del paladar anterior que se conoce como foramen incisivo y que por lo general se encuentra inmediatamente debajo de la papila incisiva. Este canal se divide en dos canalículos en su camino hacia la cavidad nasal y terminan en el piso nasal en ambos lados del tabique (Radlanski et al., 2004).

Radiográficamente el CNP aparece como un área radiolúcida entre dos líneas radiopacas paralelas que representan los límites del canal y que se extienden desde el piso de la cavidad nasal hasta el foramen incisivo (Neves et al., 2013). El foramen incisivo se identifica radiográficamente como una imagen radiolúcida de forma circular limitada por un margen radiopaco (Mraiwa et al., 2004); la separación incompleta dentro del canal, así como la superposición de la espina nasal anterior sobre el foramen incisivo pueden dar una imagen radiográfica en forma de "corazón" (Song et al., 2009), mientras que desde el plano sagital presenta una forma de "reloj de arena" o de "banana" (Mardinger et al., 2008).

En la última década, la tomografía computarizada de haz cónico (CBCT por sus siglas en inglés) se ha convertido en una herramienta útil para el diagnóstico y estudio de las estructuras del macizo facial (Bou Serhal et al., 2000). La CBCT se considera una de las mejores opciones para la visualización tridimensional de estructuras que suelen ser difíciles de evaluar mediante radiografías convencionales (Asaumi et al., 2010). El CBCT presenta gran potencial diagnóstico con la ventaja de requerir bajas dosis de radiación (Dula et al., 2014), por lo tanto la evaluación mediante CBCT debe considerarse como un método imagenológico de diagnóstico básico. 
El propósito de este estudio fue determinar la morfología y dimensiones promedio del canal nasopalatino en la población mexicana mediante tomografía computarizada de haz cónico (CBCT).

\section{MATERIAL Y MÉTODO}

Este estudio se realizó en la Maestría de Ciencias Odontológicas de la Facultad de Estomatología de la Universidad Autónoma de San Luis Potosí, México. El tipo de muestreo fue aleatorio y se incluyeron pacientes mexicanos con un rango de edad de entre 18-60 años a los que se hubiera solicitado CBCT con fines diagnósticos y clínicos. Todos los pacientes que participaron aceptaron ser parte de este estudio de manera voluntaria. El estudio fue aprobado por el comité de ética de la Facultad de Estomatología de la Universidad Autónoma de San Luis Potosí.

Las CBCT fueron adquiridas mediante un tomógrafo Kodak 9000 3D system. Se analizaron CBCT de 120 pacientes mediante cortes coronales, transversales y sagitales empleando el software CDS 3D imaging Software Ink. Empleando la clasificación de Bornstein las imágenes fueron divididas en tres grupos (Fig. 1): (grupo A) CNP con un solo canal; (grupo B) CNP con dos canales paralelos; (grupo C) CNP de tipo Y con una vía oral palatina y dos o más

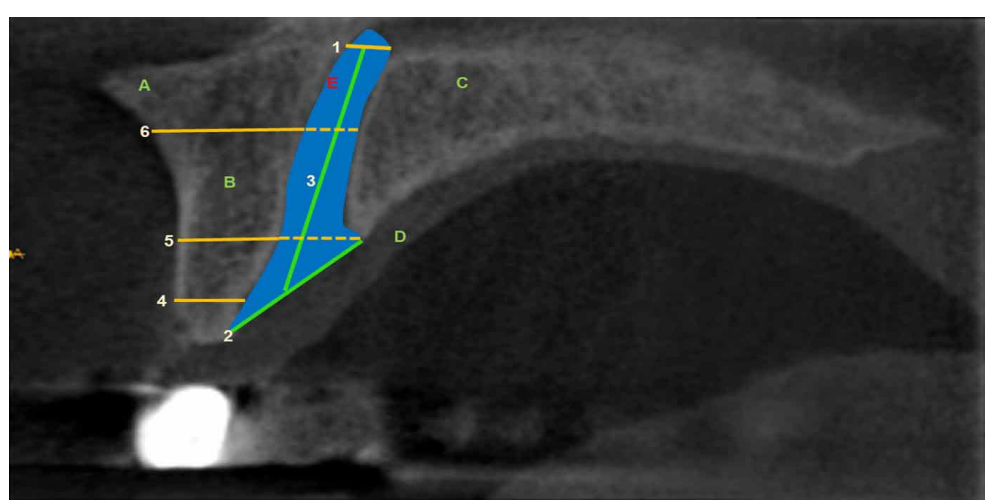

Fig. 1. Clasificación de las variaciones anatómicas del canal nasopalatino. A. Tipo A: un solo conducto; B. Tipo B: dos conductos paralelos; y C. Tipo C: variaciones en forma "Y" del canal con dos o más salidas.
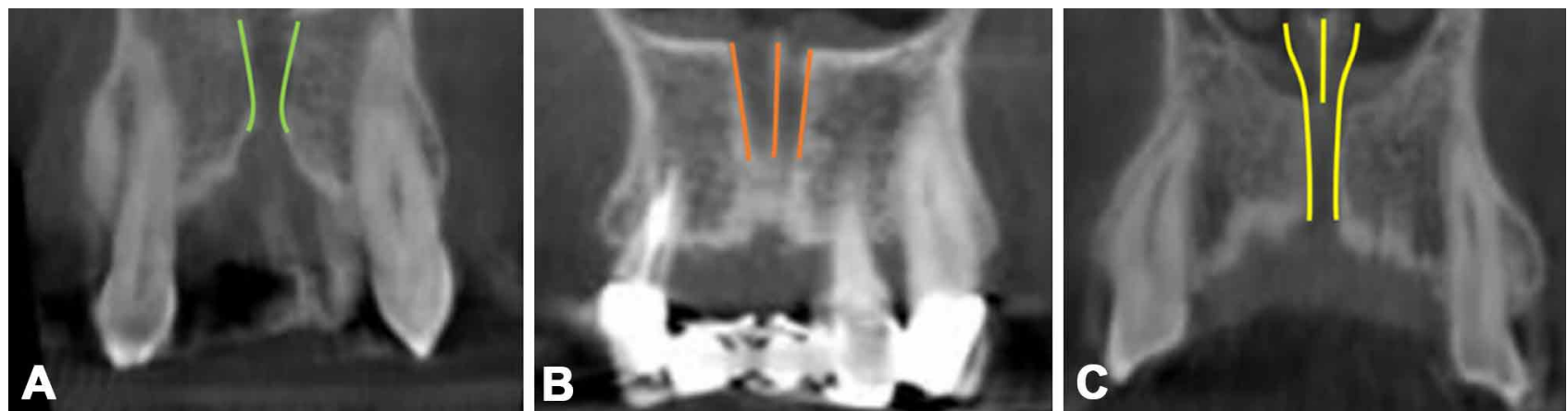

Fig. 2. Corte sagital de CNP. 1) Diámetro foramen nasal. 2) Diámetro foramen incisivo. 3) Longitud Canal nasopalatino. 4) Inferior. 5) Medio. 6) Superior. A) Espina nasal anterior. B) Proceso alveolar. C) Procesos palatinos. D) Mucosa palatina. E) Canal nasopalatino. 
ROCHA CASTILlO, A.; GARCÍA CORTES, J. O.; GUTIÉRREZ CANTÚ, F. J.; RUIZ FLORES, A.; MUÑOZ-RUÍZ, A. I.; LÓPEZ RAMÍREZ, J. C. \& MARIEL CÁRDENAS, J. Estudio morfológico de canales nasopalatinos en población mexicana mediante tomografía computarizada de haz cónico. Int. J. Morphol., 37(4):1272-1279, 2019.

Tabla I. Estadística descriptiva de cada uno de los grupos de estudio para cada variable. Incluye media, error de la media, desviación estándar, valor mínimo, mediana y valor máximo.

\begin{tabular}{|c|c|c|c|c|c|c|c|c|}
\hline Variable & $\begin{array}{c}\text { Clasificación } \\
\text { Bornstein }\end{array}$ & Sexo & Media & $\begin{array}{c}\text { Error de la } \\
\text { media }\end{array}$ & Desv.Est. & Mínimo & Mediana & Máximo \\
\hline \multirow{6}{*}{$\begin{array}{l}\text { Longitud del canal } \\
\text { nasopalatino }\end{array}$} & \multirow[t]{2}{*}{ A } & Hombres & 16,250 & 0,984 & 3,111 & 10,700 & 17,250 & 21,100 \\
\hline & & Mujeres & 14,400 & 0,827 & 2,614 & 10,400 & 13,850 & 19,500 \\
\hline & \multirow[t]{2}{*}{ B } & Hombres & 15,320 & 0,593 & 1,874 & 12,100 & 15,600 & 17,300 \\
\hline & & Mujeres & 11,48 & 1,22 & 3,85 & 4,80 & 12,30 & 15,00 \\
\hline & \multirow[t]{2}{*}{$\mathrm{C}$} & Hombres & 17,260 & 0,931 & 2,946 & 13,400 & 17,000 & 20,400 \\
\hline & & Mujeres & 12,160 & 0,764 & 2,416 & 9,200 & 11,900 & 16,800 \\
\hline \multirow{6}{*}{$\begin{array}{l}\text { Diámetro del foramen } \\
\text { nasal corte sagital }\end{array}$} & \multirow[t]{2}{*}{ A } & Hombres & 6,620 & 0,911 & 2,881 & 3,400 & 6,450 & 13,300 \\
\hline & & Mujeres & 5,850 & 0,943 & 2,983 & 2,100 & 5,800 & 11,700 \\
\hline & \multirow[t]{2}{*}{ B } & Hombres & 4,020 & 0,236 & 0,747 & 3,300 & 3,700 & 5,100 \\
\hline & & Mujeres & 4,520 & 0,932 & 2,946 & 1,500 & 3,600 & 9,600 \\
\hline & \multirow[t]{2}{*}{$\mathrm{C}$} & Hombres & 8,350 & 0,840 & 2,657 & 5,800 & 7,550 & 12,600 \\
\hline & & Mujeres & 5,59 & 1,27 & 4,01 & 0,90 & 4,10 & 11,20 \\
\hline \multirow{6}{*}{$\begin{array}{l}\text { Diámetro del foramen } \\
\text { palatino corte sagital }\end{array}$} & \multirow[t]{2}{*}{ A } & Hombres & 6,89 & 1,07 & 3,38 & 1,30 & 7,25 & 13,80 \\
\hline & & Mujeres & 7,36 & 1,03 & 3,26 & 2,00 & 7,90 & 13,00 \\
\hline & \multirow[t]{2}{*}{ B } & Hombres & 9,200 & 0,498 & 1,573 & 7,600 & 8,900 & 12,000 \\
\hline & & Mujeres & 5,240 & 0,929 & 2,937 & 1,600 & 6,500 & 8,100 \\
\hline & \multirow[t]{2}{*}{$\mathrm{C}$} & Hombres & 9,55 & 1,53 & 4,83 & 4,90 & 7,50 & 17,90 \\
\hline & & Mujeres & 7,110 & 0,544 & 1,719 & 4,400 & 7,500 & 9,000 \\
\hline \multirow{6}{*}{$\begin{array}{l}\text { Diámetro de parte } \\
\text { coronal de canal en corte } \\
\text { sagital }\end{array}$} & \multirow[t]{2}{*}{ A } & Hombres & 3,280 & 0,346 & 1,095 & 1,200 & 3,100 & 4,700 \\
\hline & & Mujeres & 2,540 & 0,337 & 1,066 & 1,600 & 2,200 & 5,300 \\
\hline & \multirow[t]{2}{*}{ B } & Hombres & 3,780 & 0,300 & 0,948 & 2,100 & 4,100 & 4,700 \\
\hline & & Mujeres & 3,660 & 0,465 & 1,471 & 2,400 & 2,900 & 6,300 \\
\hline & \multirow[t]{2}{*}{$\mathrm{C}$} & Hombres & 3,290 & 0,523 & 1,654 & 1,900 & 2,800 & 7,000 \\
\hline & & Mujeres & 3,460 & 0,468 & 1,481 & 1,600 & 3,450 & 5,500 \\
\hline \multirow{6}{*}{$\begin{array}{l}\text { Diámetro de parte media } \\
\text { de canal en corte sagital }\end{array}$} & A & Hombres & 1,940 & 0,275 & 0,868 & 0,800 & 2,000 & 3,500 \\
\hline & & Mujeres & 2,080 & 0,290 & 0,917 & 0,500 & 2,000 & 3,300 \\
\hline & $\mathrm{B}$ & Hombres & 1,660 & 0,316 & 0,999 & 0,300 & 1,500 & 3,200 \\
\hline & & Mujeres & 1,720 & 0,195 & 0,616 & 1,100 & 1,600 & 2,400 \\
\hline & $\mathrm{C}$ & Hombres & 2,630 & 0,288 & 0,912 & 1,200 & 2,650 & 4,000 \\
\hline & & Mujeres & 2,220 & 0,307 & 0,969 & 1,300 & 2,100 & 4,000 \\
\hline Diámetro de parte apical & $\mathrm{A}$ & Hombres & 3,180 & 0,576 & 1,821 & 1,200 & 2,750 & 6,200 \\
\hline de canal en corte sagital & & Mujeres & 4,030 & 0,839 & 2,653 & 1,100 & 3,400 & 10,700 \\
\hline & $\mathrm{B}$ & Hombres & 4,100 & 0,552 & 1,746 & 2,100 & 4,000 & 6,600 \\
\hline & & Mujeres & 3,020 & 0,353 & 1,116 & 1,600 & 2,800 & 4,700 \\
\hline & $\mathrm{C}$ & Hombres & 4,200 & 0,493 & 1,560 & 1,900 & 3,700 & 6,600 \\
\hline & & Mujeres & 3,300 & 0,531 & 1,679 & 0,800 & 3,550 & 5,600 \\
\hline Diámetro de parte & A & Hombres & 3,540 & 0,609 & 1,926 & 1,000 & 3,250 & 6,400 \\
\hline coronal de canal en corte & & Mujeres & 3,110 & 0,457 & 1,445 & 0,900 & 3,300 & 5,300 \\
\hline coronal & B & Hombres & 4,400 & 0,283 & 0,894 & 3,200 & 4,400 & 5,800 \\
\hline & & Mujeres & 3,625 & 0,499 & 1,411 & 1,600 & 3,800 & 5,300 \\
\hline & $\mathrm{C}$ & Hombres & 5,52 & 1,12 & 3,53 & 0,40 & 4,60 & 11,30 \\
\hline & & Mujeres & 3,760 & 0,523 & 1,655 & 2,100 & 3,350 & 6,800 \\
\hline Diámetro de parte media & A & Hombres & 2,630 & 0,290 & 0,918 & 1,100 & 2,700 & 4,400 \\
\hline de canal en corte coronal & & Mujeres & 3,250 & 0,434 & 1,371 & 1,100 & 3,700 & 4,800 \\
\hline & $\mathrm{B}$ & Hombres & 4,260 & 0,458 & 1,448 & 2,600 & 4,200 & 6,000 \\
\hline & & Mujeres & 3,720 & 0,238 & 0,752 & 2,600 & 3,700 & 4,800 \\
\hline & $\mathrm{C}$ & Hombres & 3,300 & 0,427 & 1,349 & 2,100 & 3,300 & 6,600 \\
\hline & & Mujeres & 3,660 & 0,468 & 1,479 & 1,600 & 3,450 & 5,800 \\
\hline Diámetro de parte apical & A & Hombres & 2,660 & 0,409 & 1,295 & 1,200 & 2,350 & 5,100 \\
\hline de canal en corte coronal & & Mujeres & 3,040 & 0,426 & 1,346 & 0,900 & 2,950 & 5,500 \\
\hline & B & Hombres & 3,760 & 0,392 & 1,241 & 2,100 & 3,500 & 5,600 \\
\hline & & Mujeres & 4,200 & 0,212 & 0,670 & 3,100 & 4,400 & 5,000 \\
\hline & $\mathrm{C}$ & Hombres & 6,93 & 1,13 & 3,56 & 3,50 & 6,45 & 15,20 \\
\hline & & Mujeres & 4,740 & 0,613 & 1,940 & 2,200 & 4,800 & 7,400 \\
\hline
\end{tabular}


ROCHA CASTILlO, A.; GARCÍA CORTES, J. O.; GUTIÉRREZ CANTÚ, F. J.; RUIZ FLORES, A.; MUÑOZ-RUÍZ, A. I.; LÓPEZ RAMÍREZ, J. C. \& MARIEL CÁRDENAS, J. Estudio morfológico de canales nasopalatinos en población mexicana mediante tomografía computarizada de haz cónico. Int. J. Morphol., 37(4):1272-1279, 2019.

\begin{tabular}{|c|c|c|c|c|c|c|c|c|}
\hline Variable & $\begin{array}{c}\text { Clasificación } \\
\text { Bornstein }\end{array}$ & Sexo & Media & $\begin{array}{c}\text { Error de la } \\
\text { media }\end{array}$ & Desv.Est. & Mínimo & Medi ana & Máximo \\
\hline \multirow{6}{*}{$\begin{array}{l}\text { Grosor óseo desde el margen anterior } \\
\text { del foramen }\end{array}$} & \multirow[t]{2}{*}{ A } & Hombres & 6,460 & 0,536 & 1,695 & 2,300 & 7,000 & 8,200 \\
\hline & & Mujeres & 6,940 & 0,413 & 1,307 & 5,600 & 6,600 & 9,600 \\
\hline & \multirow[t]{2}{*}{ B } & Hombres & 6,960 & 0,453 & 1,432 & 5,200 & 6,800 & 8,600 \\
\hline & & Mujeres & 5,800 & 0,623 & 1,970 & 3,400 & 7,000 & 7,900 \\
\hline & \multirow[t]{2}{*}{$\mathrm{C}$} & Hombres & 4,730 & 0,754 & 2,384 & 1,900 & 4,650 & 8,700 \\
\hline & & Mujeres & 7,630 & 0,369 & 1,168 & 5,800 & 7,550 & 9,100 \\
\hline \multirow{6}{*}{$\begin{array}{l}\text { Grosor óseo desde pared anterior del } \\
\text { canal en relación con margen } \\
\text { posterior del foramen }\end{array}$} & \multirow[t]{2}{*}{ A } & Hombres & 7,120 & 0,646 & 2,044 & 3,300 & 7,450 & 11,100 \\
\hline & & Mujeres & 6,960 & 0,578 & 1,828 & 5,000 & 6,850 & 11,200 \\
\hline & \multirow[t]{2}{*}{ B } & Hombres & 9,200 & 0,764 & 2,418 & 6,100 & 9,000 & 12,900 \\
\hline & & Mujeres & 9,040 & 0,970 & 3,067 & 3,700 & 10,000 & 12,000 \\
\hline & \multirow[t]{2}{*}{$\mathrm{C}$} & Hombres & 5,780 & 0,645 & 2,039 & 3,100 & 5,650 & 9,100 \\
\hline & & Mujeres & 8,090 & 0,667 & 2,111 & 5,500 & 8,600 & 11,900 \\
\hline \multirow{6}{*}{$\begin{array}{l}\text { Grosor óseo desde pared anterior del } \\
\text { canal en su parte media }\end{array}$} & \multirow[t]{2}{*}{ A } & Hombres & 6,760 & 0,721 & 2,280 & 1,500 & 7,650 & 9,500 \\
\hline & & Mujeres & 6,700 & 0,499 & 1,577 & 4,300 & 6,800 & 9,100 \\
\hline & \multirow[t]{2}{*}{ B } & Hombres & 7,960 & 0,447 & 1,412 & 6,300 & 7,900 & 10,000 \\
\hline & & Mujeres & 7,280 & 0,504 & 1,595 & 4,400 & 7,500 & 8,700 \\
\hline & \multirow[t]{2}{*}{$\mathrm{C}$} & Hombres & 5,770 & 0,642 & 2,031 & 2,900 & 6,400 & 8,400 \\
\hline & & Mujeres & 7,460 & 0,380 & 1,203 & 6,200 & 7,400 & 9,500 \\
\hline \multirow{6}{*}{$\begin{array}{l}\text { Densidad ósea de pared anterior de } \\
\text { canal nasopalatino }\end{array}$} & \multirow[t]{2}{*}{ A } & Hombres & 752 & 109 & 345 & 135 & 717 & 1345 \\
\hline & & Mujeres & 786,1 & 72,1 & 228,0 & 338,0 & 838,0 & 1078,0 \\
\hline & \multirow[t]{2}{*}{ B } & Hombres & 781,2 & 74,7 & 236,1 & 524,0 & 780,0 & 1067,0 \\
\hline & & Mujeres & 745,4 & 50,6 & 160,0 & 501,0 & 721,0 & 912,0 \\
\hline & \multirow[t]{2}{*}{$\mathrm{C}$} & Hombres & 723,1 & 48,3 & 152,8 & 472,0 & 724,0 & 962,0 \\
\hline & & Mujeres & 802,8 & 55,3 & 174,9 & 539,0 & 855,5 & 985,0 \\
\hline \multirow{6}{*}{$\begin{array}{l}\text { Densidad ósea de pared posterior del } \\
\text { canal nasopalatino }\end{array}$} & \multirow[t]{2}{*}{ A } & Hombres & 607,7 & 81,0 & 256,3 & 128,0 & 626,5 & 972,0 \\
\hline & & Mujeres & 826,3 & 91,1 & 288,1 & 418,0 & 818,0 & 1186,0 \\
\hline & \multirow[t]{2}{*}{ B } & Hombres & 826,3 & 91,1 & 288,1 & 418,0 & 818,0 & 1186,0 \\
\hline & & Mujeres & 774,6 & 61,6 & 194,6 & 482,0 & 892,0 & 945,0 \\
\hline & \multirow[t]{2}{*}{$\mathrm{C}$} & Hombres & 692 & 107 & 340 & 121 & 609 & 1260 \\
\hline & & Mujeres & 740,2 & 52,5 & 166,1 & 502,0 & 813,0 & 942,0 \\
\hline \multirow{6}{*}{$\begin{array}{l}\text { Densidad ósea de pared izquierda de } \\
\text { canal nasopalatino }\end{array}$} & \multirow[t]{2}{*}{ A } & Hombres & 632 & 110 & 349 & 381 & 484 & 1518 \\
\hline & & Mujeres & 688 & 101 & 320 & 251 & 695 & 1179 \\
\hline & \multirow[t]{2}{*}{ B } & Hombres & 919,8 & 98,8 & 312,4 & 537,0 & 859,0 & 1427,0 \\
\hline & & Mujeres & 764 & 114 & 361 & 310 & 841 & 1195 \\
\hline & $\mathrm{C}$ & Hombres & 642,2 & 58,2 & 184,2 & 493,0 & 557,5 & 994,0 \\
\hline & & Mujeres & 758,4 & 59,6 & 188,3 & 497,0 & 784,5 & 1009,0 \\
\hline Densidad ósea de pared derecha de & A & Hombres & 558,4 & 49,2 & 155,7 & 355,0 & 583,5 & 878,0 \\
\hline canal nasopalatino & & Mujeres & 851 & 109 & 344 & 291 & 806 & 1415 \\
\hline & B & Hombres & 862,6 & 97,6 & 308,5 & 398,0 & 1021,0 & 1207,0 \\
\hline & & Mujeres & 780,0 & 89,7 & 283,7 & 423,0 & 941,0 & 1060,0 \\
\hline & $\mathrm{C}$ & Hombres & 631,9 & 67,8 & 214,4 & 342,0 & 673,0 & 981,0 \\
\hline & & Mujeres & 835,0 & 64,9 & 205,3 & 578,0 & 910,0 & 1097,0 \\
\hline
\end{tabular}

\section{RESULTADOS}

Se identificaron diferencias estadísticamente significativas en las siguientes variables evaluadas en hombres: Diámetro del foramen nasal corte sagital del tipo A comparado con el tipo $\mathrm{B}(\mathrm{p}=\leq 0,0089)$ y del tipo $\mathrm{B}$ comparado con el tipo $\mathrm{C}$ ( $\mathrm{p}=\leq 0,0002)$; Diámetro del foramen palatino corte sagital del tipo A comparado con el tipo B ( $\mathrm{p}=\leq 0,011)$; Diámetro de parte media de canal en corte sagital del tipo A comparado con el tipo $\mathrm{C}(\mathrm{p}=\leq 0,0357)$ y del tipo B comparado con el tipo $\mathrm{C}(\mathrm{p}=\leq 0,0448)$; Diámetro de parte apical de canal en corte sagital del tipo A comparado con el tipo C $(\mathrm{p}=\leq 0,0386)$; Diámetro de parte media de canal en corte coronal del tipo A comparado con el tipo B $(\mathrm{p}=\leq 0,0306)$; Diámetro de parte apical de canal en corte coronal del tipo A comparado con el tipo B ( $\mathrm{p}=\leq 0,0372)$ y del tipo A comparado con el tipo $C$ ( $p=\leq 0,0013)$; Grosor óseo desde el margen anterior del foramen del tipo $\mathrm{B}$ comparado con el tipo $\mathrm{C}$ $(\mathrm{p}=\leq 0,0254)$; Grosor óseo desde pared anterior del canal en relación con margen posterior del foramen del grupo A com- 
parado con el tipo $\mathrm{B}(\mathrm{p}=\leq 0,0500)$ y del tipo $\mathrm{B}$ comparado con el tipo $\mathrm{C}(\mathrm{p}=\leq 0,0045)$; Grosor óseo desde pared anterior del canal en su parte media del tipo B comparado con el tipo C (0,0371); Densidad ósea de pared posterior del canal nasopalatino del tipo A comparado con el tipo B $(\mathrm{p}=\leq 0,0170)$; Densidad ósea de pared izquierda de canal nasopalatino del tipo A comparado con el tipo B $(p=\leq 0,0170)$ y del tipo B comparado con el tipo $C$ ( $p=\leq 0,0306)$; Densidad ósea de pared derecha de canal nasopalatino del tipo A comparado con el tipo $B(p=\leq 0,0255)$ (Tabla II).

Se identificaron diferencias estadísticamente significativas en las siguientes variables evaluadas en mujeres: Longitud del canal nasopalatino del grupo A comparado con el tipo $\mathrm{C}$ ( $\mathrm{p}=\leq 0$,0492); Diámetro del formen na- sal en corte sagital del tipo A comparado con el tipo B $(\mathrm{p}=\leq 0,0167)$; Diámetro de parte coronal de canal en corte coronal del tipo A comparado con el tipo B ( $\mathrm{p}=\leq 0,0254)$; Grosor óseo desde el margen anterior del foramen del tipo B comparado con el tipo C ( $\mathrm{p}=\leq 0,0439$ ); Grosor óseo desde pared anterior del canal en relación con margen posterior del foramen del tipo A comparado con el tipo B $(\mathrm{p}=\leq 0,0451)$ (Tabla III).

Se identificaron diferencias estadísticamente significativas en las siguientes variables evaluadas entre hombres y mujeres: Longitud del canal nasopalatino del tipo B $(p=\leq 0,0044)$ y $C(p=\leq 0,0017)$; Diámetro del foramen incisivo corte sagital del grupo $\mathrm{B}(\mathrm{p}=\leq 0,0016)$; Diámetro de parte nasal de canal en corte sagital del tipo A

Tabla II. Comparación de grupos de estudio por clasificación Bornstein para cada una de las variables en hombres.

\begin{tabular}{|c|c|c|c|}
\hline \multirow[b]{2}{*}{ Variables } & \multicolumn{3}{|c|}{$\begin{array}{l}\text { Comparación de grupos por } \\
\text { clasificación B ornstein }\end{array}$} \\
\hline & $\mathrm{A}$ vs B & A vs $\mathrm{C}$ & $\mathrm{B}$ vs $\mathrm{C}$ \\
\hline Longitud del canal nasopalatino & 0,2716 & 0,6497 & 0,1602 \\
\hline Diámetro del foramen nasal corte sagital & $0,0089 * *$ & 0,1854 & 0,0002 \\
\hline Diámetro del foramen palatino corte sagital & $0,0111 *$ & 0,3436 & 0,3834 \\
\hline Diámetro de parte coronal de canal en corte sagital & 0,4258 & 0,6769 & 0,3429 \\
\hline Diámetro de parte media de canal en corte sagital & 0,4717 & $0,0357^{*}$ & $0,0448 *$ \\
\hline Diámetro de parte apical de canal en corte sagital & 0,2107 & $0,0386^{*}$ & 0,9697 \\
\hline Diámetro de parte coronal de canal en corte coronal & 0,3064 & 0,2725 & 0,6768 \\
\hline Diámetro de parte media de canal en corte coronal & $0,0306^{*}$ & 0,2237 & 0,1594 \\
\hline Diámetro de parte apical de canal en corte coronal & $0,0372 *$ & $0,0013 * *$ & 0,0109 \\
\hline Grosor óseo desde el margen anterior del foramen & 0,7901 & 0,0749 & $0,0254 *$ \\
\hline Grosor óseo desde pared anterior del canal en relación con margen post erior del foramen & $0,0500 *$ & 0,2260 & $0,0045 * *$ \\
\hline Grosor óseo desde pared anterior del canal en su parte media & 0,2716 & 0,2723 & $0,0371 *$ \\
\hline Densidad ósea de pared anterior de canal nasopalatino & 0,9096 & 0,9698 & 0,5697 \\
\hline Densidad ósea de pared posterior del canal nasopalatino & $0,0170 *$ & 0,7335 & 0,3062 \\
\hline Densidad ósea de pared izquierda de canal nasopalatino & $0,0170 *$ & 0,3071 & $0,0306^{*}$ \\
\hline Densidad ósea de pared derecha de canal nasopalatino & $0,0255^{*}$ & 0,4723 & 0,0749 \\
\hline
\end{tabular}

Tabla III. Comparación de grupos de estudio por clasificación Bornstein para cada una de las variables en mujeres.

\begin{tabular}{lccc}
\hline & \multicolumn{3}{c}{$\begin{array}{c}\text { Comparación de grupos por } \\
\text { clasificación Bornstein }\end{array}$} \\
\cline { 2 - 4 } Variables & A vs B & A vs C & B vs C \\
\hline Longitud del canal nasopalatino & 0,1397 & $0,0492^{*}$ & 0,9697 \\
Diámetro del foramen nasal corte sagital & 0,2716 & 0,7334 & 0,6767 \\
Diámetro del foramen palatino corte sagital & 0,1396 & 1,0000 & 0,1602 \\
Diámetro de parte coronal de canal en corte sagital & $0,0167^{*}$ & 0,2251 & 0,9696 \\
Diámetro de parte media de canal en corte sagital & 0,3033 & 1,0000 & 0,4686 \\
Diámetro de parte apical de canal en corte sagital & 0,4718 & 0,7909 & 0,5188 \\
Diámetro de parte coronal de canal en corte coronal & 0,3471 & 0,4044 & 0,8931 \\
Diámetro de parte media de canal en corte coronal & 0,6762 & 0,6757 & 0,5683 \\
Diámetro de parte apical de canal en corte coronal & $0,0254 *$ & 0,1116 & 0,3427 \\
Grosor óseo desde el margen anterior del foramen & 0,9092 & 0,1293 & $0,0439 *$ \\
Grosoróseo desde pared anterior del canal en relación con margen & $0,0451^{*}$ & 0,1976 & 0,2394 \\
posterior del foramen & & & 0,4695 \\
Grosoróseo desde pared anterior del canal en su parte media & 0,2700 & 0,4483 & 0,4713 \\
Densidad ósea de pared anterior de canal nasopalatino & 0,5700 & 0,9095 & 0,3060 \\
Densidad ósea de pared posterior del canal nasopalatino & 0,7910 & 0,5196 & 0,7907 \\
Densidad ósea de pared izquierda de canal nasopalatino & 0,4718 & 0,5201 & 0,7907 \\
Densidad ósea de pared derecha de canal nasopalatino & 0,5700 & 0,9698 & \\
\hline
\end{tabular}


ROCHA CASTILLO, A.; GARCÍA CORTES, J. O.; GUTIÉRREZ CANTÚ, F. J.; RUIZ FLORES, A.; MUÑOZ-RUÍZ, A. I.; LÓPEZ RAMÍREZ, J. C. \& MARIEL CÁRDENAS, J. Estudio morfológico de canales nasopalatinos en población mexicana mediante tomografía computarizada de haz cónico. Int. J. Morphol., 37(4):1272-1279, 2019.

$(\mathrm{p}=\leq 0,0452)$; Grosor óseo desde el margen anterior del foramen del tipo $\mathrm{C}(\mathrm{p}=\leq 0,0072)$; Grosor óseo desde pared anterior del canal en relación con margen posterior del foramen del tipo $\mathrm{C}(\mathrm{p}=\leq 0,0432)$; Densidad ósea de pared derecha de canal nasopalatino del tipo A $(\mathrm{p}=\leq 0,0376)$ y $\mathrm{C}(\mathrm{p}=\leq 0,0384)$ (Tabla IV).

Tabla IV. Comparación de grupos de estudio por clasificación Bornstein para cada una de las variables entre hombres y mujeres.

\begin{tabular}{|c|c|c|c|}
\hline \multirow[b]{2}{*}{ Variables } & \multicolumn{3}{|c|}{$\begin{array}{l}\text { Comparación de grupos por } \\
\text { clasificación Bornstein }\end{array}$} \\
\hline & A & $\mathrm{B}$ & $\mathrm{C}$ \\
\hline Longitud del canal nasopalatino & 0,1617 & $0,0044 * *$ & $0,0017 * *$ \\
\hline Diámetro del foramen nasal corte sagital & 0,5205 & 0,7899 & 0,1204 \\
\hline Diámetro del foramen palatino corte sagital & 0,7335 & $0,0016^{* *}$ & 0,4265 \\
\hline Diámetro de parte coronal de canal en corte sagital & $0,0452 *$ & 0,4697 & 0,7332 \\
\hline Diámetro de parte media de canal en corte sagital & 0,7615 & 0,9088 & 0,3058 \\
\hline Diámetro de parte apical de canal en corte sagital & 0,4722 & 0,1842 & 0,2888 \\
\hline Diámetro de parte coronal de canal en corte coronal & 0,6499 & 0,1647 & 0,2254 \\
\hline Diámetro de parte media de canal en corte coronal & 0,2114 & 0,3814 & 0,4702 \\
\hline Diámetro de parte apical de canal en corte coronal & 0,3827 & 0,3814 & 0,1612 \\
\hline Grosor óseo desde el margen anterior del foramen & 0,6223 & 0,3042 & $0,0072 * *$ \\
\hline Grosor óseo desde pared anterior del canal en relación con margen posterior del foramen & 0,5452 & 0,9094 & $0,0432 *$ \\
\hline Grosor óseo desde pared anterior del canal en su parte media & 0,6230 & 0,4697 & 0,1180 \\
\hline Densidad ósea de pared anterior de canal nasopalatino & 0,6232 & 0,4710 & 0,3066 \\
\hline Densidad ósea de pared posterior del canal nasopalatino & 0,1620 & 0,4727 & 0,9698 \\
\hline Densidad ósea de pared izquierda de canal nasopalatino & 0,5966 & 0,4710 & 0,2114 \\
\hline Densidad ósea de pared derecha de canal nasopalatino & $0,0376^{*}$ & 0,4710 & $0,0384^{*}$ \\
\hline
\end{tabular}

\section{DISCUSIÓN}

En este estudio se evaluaron las variantes y diferencias morfológicas del CNP en población mexicana. Los datos obtenidos corroboran información previamente reportada y proporciona nuevos datos morfológicos referentes a esta zona anatómica. Diversos estudios (Thakur, et al., 2013; Hakbilen \& Magat, 2018; Costa et al., 2019; Cazar Almache et al., 2019) han reportado previamente la presencia de estas variaciones, sin embargo no existían reportes previos en la población mexicana. De acuerdo con los datos obtenidos se puede determinar que existen variaciones morfológicas considerables del CNP entre los individuos incluidos en el estudio, por lo que se recomienda realizar un análisis minucioso y exhaustivo del área si se desea realizar alguna intervención o procedimiento quirúrgico en la zona.

De acuerdo al análisis realizado se puede determinar que la longitud del CNP es mayor en hombres que en mujeres; en el caso del diámetro nasal, el hombre presenta mayor dimensión, con excepción de la morfología tipo $\mathrm{C}$ de Bornstein, en donde la mujer presenta un diámetro mayor; en el caso del diámetro incisivo, el hombre presenta mayor dimensión, con excepción de la morfología tipo A de Bornstein, en donde la mujer presenta un diámetro mayor; en relación a la distancia que va del margen anterior del
CNP a la tabla ósea vestibular, la mujer presenta un grosor mayor al hombre, con excepción de la morfología tipo B en donde el del hombre es mayor.

Los datos aquí reportados corroboran la información previamente reportada por Thakur et al., en su estudio reportan que existen diferencias significativas en la longitud del CNP entre los individuos, siendo mayor en los hombre con una media de $10,96 \mathrm{~mm}$ y en mujeres con una media de $9,20 \mathrm{~mm}$, esta diferencia asociada al sexo se puede atribuir a la diferencia en las dimensiones craneales entre ambos.

Hakbilen \& Magat analizaron 619 CBCT en población turca de entre 17-86 años. De acuerdo con los datos se concluyó que existe una gran variabilidad en términos de morfología y dimensiones del CNP, en donde la forma de reloj de arena es la más común $(26,17 \%)$, este tipo de forma correspondería con la clasificación tipo A y/o C según Bornstein, nuestro estudio corrobora estos datos si se consideran ambos tipos morfológicos como uno solo. Por otro lado, Costa et al. evaluaron 132 CBCT tanto en hombres como mujeres en población brasileña con rangos de edad de entre 21-60 años; se reporta que los pacientes mayores de 50 años presentan un canal nasopalatino significativamente 
mayor, además de que en relación al tipo de anatomía la salida única del canal era la más común y en menor frecuencia dos canales paralelos. Estos datos coinciden con los datos aquí obtenidos, en donde si se considera que los tipos A y C usualmente presentan una única salida.

Bornstein et al. determinaron que el tipo de morfología del canal nasopalatino influye en el diámetro del foramen incisivo, en donde el diámetro más ancho se asocia al tipo A, seguida del tipo $\mathrm{B}$ y por último el tipo $\mathrm{C}$; estos datos difieren a los del presente estudio, donde se observó que en hombres el grupo con mayor diámetro de foramen incisivo es el tipo B, continuando con el tipo A y por último con el tipo $\mathrm{C}$; esta diferencia en los datos se puede deber que el tipo de población difiere entre los estudios, además se debe considerar que el diámetro del foramen y canal están directamente relacionado con el tipo y volumen de estructuras que este alberga.

Bornstein et al. reportaron que la tabla vestibular ósea está afectada por el género de la persona y es independiente a las características geométricas y clasificación; los datos reportados por el estudio de Khojastepour et al. (2017) concuerdan con lo ya mencionado, en donde el grosor óseo de la pared anterior del canal en relación con margen posterior al foramen de los hombres $(7,36 \pm 1,45 \mathrm{~mm})$ es mayor que en el de las mujeres $(6,78 \pm 1,27 \mathrm{~mm})$; estos datos concuerdan con lo reportado en nuestro estudio, sin embargo se identifica diferencia significativa en la clasificación tipo $\mathrm{C}$, en donde el diámetro incisivo en hombres es de 5,65 mm y en mujeres $8,6 \mathrm{~mm}$ con un diferencia estadísticamente significativa $(\mathrm{p}=\leq 0,0432)$.

Thakur et al. reportaron que la mediana antero-posterior del diámetro del CNP en corte sagital es de 2,78 $( \pm 0,85)$ $\mathrm{mm}$. El promedio es a nivel de foramen nasal es de $2,86 \mathrm{~mm}$, en la parte media es de $2,32 \mathrm{~mm}$, y foramen incisivo es de $3,15 \mathrm{~mm}$, la diferencia entre hombres y mujeres, en estas medidas no se obtuvo una diferencia significativa. Nuestro estudio no concuerda con este, ya que hubo diferencia significativa en las medidas entre hombres y mujeres en el tipo B, en la parte incisal del canal, mientras que en el tipo $\mathrm{A}$ y $\mathrm{C}$ no hubo diferencias significativas en ninguno de las tres medidas; El diámetro del foramen incisivo en el tipo B es de 7,90 mm en mujeres y $8,90 \mathrm{~mm}$ hombres, con una diferencia significativa de $\mathrm{P}=\leq 0,0016$, esto se puede atribuir a las morfología del canal, ya que este cuenta con dos canales.

\section{CONCLUSIÓN}

Los resultados de este estudio mostraron diferencias estadísticas significativas entre las variables analizadas del CNP entre hombres y mujeres, por lo que se puede concluir que existe una amplia variabilidad en morfología y dimensiones del CNP en la población mexicana, es posible que la aparente diferencia en las dimensiones entre hombres y mujeres puedan ser relativas y deberse a las dimensiones del macizo facial.

Se recomienda realizar un estudio morfológico y dimensional adecuado antes de efectuar cualquier intervención quirúrgica asociada a esta área; los autores consideramos que la clasificación de Bornstein podría ser empleada como parámetro para determinar la complejidad o riesgo de comunicación con el canal al momento de realizar algún procedimiento, así mismo la clasificación puede ser de utilidad en la planificación de cualquier abordaje quirúrgico en la zona.

\section{AGRADECIMIENTOS}

El presente estudio fue realizado con el equipo de la clínica radiográfica LADEM Digital, San Luis Potosí, Mex. a quien agradecemos su apoyo en el estudio. A Edgardo Martínez Martin del Campo por su colaboración en el desarrollo de la investigación en aspectos técnicos.

ROCHA CASTILLO, A.; GARCÍA CORTES, J. O.; GUTIÉRREZ CANTÚ, F. J.; RUIZ FLORES, A.; MUÑOZRUÍZ, A. I.; LÓPEZ RAMÍREZ, J. C. \& MARIEL CÁRDE-

NAS, J. Morphological study of nasopalatine canal in Mexican population using cone bean computed tomography. Int. $J$. Morphol., 37(4):1272-1279, 2019.

SUMMARY: Certain human structures present different dimensions and morphologies in each population and individual, the nasopalatine canal being one of these. It is located in the midline of the palate, and it contains the nasopalatine nerve and the terminal branch of the nasopalatine artery. The purpose of this study was to analyze and record measurements of the nasopalatine duct in Mexican population by Cone Beam Computed Tomography (CBCT). A total of 120 CBCT coronal, transversal and sagittal views were analyzed. The data were classified according to Bornstein's parameters. The normality of the variables was determined with the Shapiro Wilk test and the statistical significance was determinate by U-Mann Whitney test. A statistically significant difference was found in the evaluated variables of the nasopalatal canal between men and women. The data obtained determined that the morphology of the nasopalatine canal is variable and a morphological and dimensional analysis before any surgical intervention related with the area is recommended.

KEY WORDS: Nasopalatine duct; Bornstein parameter's; Cone Beam Computed Tomography. 


\section{REFERENCIAS BIBLIOGRÁFICAS}

Asaumi, R.; Kawai, T.; Sato, I.; Yoshida, S. \& Yosue, T. Three-dimensional observations of the incisive canal and the surrounding bone using cone-beam computed tomography. Oral Radiol., 26(1):20-8, 2010.

Bornstein, M. M.; Balsiger, R.; Sendi, P. \& von Arx, T. Morphology of the nasopalatine canal and dental implant surgery: a radiographic analysis of 100 consecutive patients using limited cone-beam computed tomography. Clin. Oral Implants Res., 22(3):295-301, 2011.

Bou Serhal, C.; Jacobs, R.; Persoons, M.; Hermans, R. \& van Steenberghe, D. The accuracy of spiral tomography to assess bone quantity for the preoperative planning of implants in the posterior maxilla. Clin. Oral Implants Res., 11(3):242-7, 2000.

Cazar Almache, M. E.; Abril Cordero, L. M.; Palacios Vivar, D. E.; Abril Cordero, M. F. \& Sibri Quizhpe, C. B. Alteraciones anatómicas del canal nasopalatino en pacientes dentados y desdentados en el sector anterosuperior utilizando tomografía computarizada de haz cónico. Acta Odontol. Colomb., 9(1):49-57, 2019.

Costa, E. D. D.; Nejaim, Y.; Martins, L. A. C.; Peyneau, P. D.; Ambrosano, G. M. B. \& Oliveira, M. L. Morphological evaluation of the nasopalatine canal in patients with different facial profiles and ages. J. Oral Maxillofac. Surg., 77(4):721-9, 2019.

Dula, K.; Bornstein, M. M.; Buser, D.; Dagassan-Berndt, D.; Ettlin, D. A.; Filippi, A.; Gabioud, F.; Katsaros, C.; Krastl, G.; Lambrecht, J. T.; et al. SADMFR Guidelines for the Use of Cone-Beam Computed Tomography/Digital Volume Tomography Oral and maxillofacial surgery, temporomandibular joint disorders and orthodontics. Swiss Dent. J., 124(11):1170-83, 2014.

Hakbilen, S. \& Magat, G. Evaluation of anatomical and morphological characteristics of the nasopalatine canal in a Turkish population by cone beam computed tomography. Folia Morphol. (Warsz), 77(3):52735, 2018 .

Jacobs, R.; Lambrichts, I.; Liang, X.; Martens, W.; Mraiwa, N.; Adriaensens, P. \& Gelan, J. Neurovascularization of the anterior jaw bones revisited using high-resolution magnetic resonance imaging. Oral Surg. Oral Med. Oral Pathol. Oral Radio. Endod., 103(5):683-93, 2007.

Khojastepour, L.; Haghnegahdar, A. \& Keshtkar, M. Morphology and dimensions of nasopalatine canal: a radiographic analysis using cone beam computed tomography. J. Dent. (Shiraz), 18(4):244-50, 2017.

Liang, X.; Jacobs, R.; Martens, W.; Hu, Y.; Adriaensens, P.; Quirynen, M. \& Lambrichts, I. Macro- and micro-anatomical, histological and computed tomography scan characterization of the nasopalatine canal. J. Clin. Periodontol., 36(7):598-603, 2009.

Mardinger, O.; Namani-Sadan, N.; Chaushu, G. \& Schwartz-Arad, D. Morphologic changes of the nasopalatine canal related to dental implantation: a radiologic study in different degrees of absorbed maxillae. J. Periodontol., 79(9):1659-62, 2008.

Mraiwa, N.; Jacobs, R.; Van Cleynenbreugel, J.; Sanderink, G.; Schutyser, F.; Suetens, P.; van Steenberghe, D. \& Quirynen, M. The nasopalatine canal revisited using 2D and 3D CT imaging. Dentomaxillofac. Radiol., 33(6):396-402, 2004.

Neves, F. S.; Oliveira, L. K.; Ramos Mariz, A. C.; Crusoé-Rebello, I. \& de Oliveira-Santos, C. Rare anatomical variation related to the nasopalatine canal. Surg. Radiol. Anat., 35(9):853-5, 2013.

Radlanski, R. J.; Emmerich, S. \& Renz, H. Prenatal morphogenesis of the human incisive canal. Anat. Embryol. (Berl.), 208(4):265-71, 2004.

Song, W. C.; Jo, D. I.; Lee, J. Y.; Kim, J. N.; Hur, M. S.; Hu, K. S.; Kim, H. J.; Shin, C. \& Koh, K. S. Microanatomy of the incisive canal using three-dimensional reconstruction of microCT images: an ex vivo study. Oral Surg. Oral Med. Oral Pathol. Oral Radiol. Endod., 108(4):58390, 2009.

Thakur, A. R.; Burde, K.; Guttal, K. \& Naikmasur, V. G. Anatomy and morphology of the nasopalatine canal using cone-beam computed tomography. Imaging Sci. Dent., 43(4):273-81, 2013.

\author{
Dirección para correspondencia: \\ Jairo Mariel Cárdenas \\ Maestría en Ciencias Odontológicas \\ Facultad de Estomatología \\ Universidad Autónoma de San Luis Potosí \\ Av. Dr. Manuel Nava \# 2 \\ Zona Universitaria, C.P. 78290 \\ San Luis Potosí, S.L.P. \\ MÉXICO
}

Email: Ilairo@hotmail.com / llairo@yahoo.com

Recibido : 16-05-2019

Aceptado: 02-07-2019 